\title{
Pharmacist-managed Warfarin in Pediatric Cardiac Patients: Quality-improvement Study
}

\author{
Carrie Tilton ", Mary Subramanian, Lauren Wyatt, Elizabeth Woods, Derek A. Williams
}

1 Medical Center Blvd, Winston-Salem, NC, USA

Copyright $\bigcirc 2018$ by authors, all rights reserved. Authors agree that this article remains permanently open access under the terms of the Creative Commons Attribution License 4.0 International License

\begin{abstract}
Objective: To compare the efficacy of warfarin management by a pharmacist-managed group to a cardiologist-managed group (usual care) in an outpatient pediatric population. Methods: Fourteen patients with an international normalized ratio (INR) goal determined by a cardiologist were included. The pharmacist-managed group included twelve patients. There were seven patients in the cardiologist-managed group. Prior to the transition to a pharmacist-managed service in September 2014, cardiologists managed anticoagulation. For the cardiologist-managed group, INR values were recorded for six months. Following a washout period of three months, INR values were obtained for the pharmacist-managed group for the subsequent six months. Patients were included in each analysis if they were followed for more than 3 months. A protocol guided dose adjustments, recommended monitoring, and allowed for pharmacist-managed warfarin. Results: The median number of INR tests per patient was not significantly lower in the cardiologist-managed group compared to the pharmacist-managed group (28 versus 36 measurements, $\mathrm{p}=0.06$ ). The median percentage of time in therapeutic range using the Rosendaal method was not significantly higher in the pharmacist-managed group compared to the cardiologist-managed group (55.5\% versus $54 \%, \mathrm{p}=0.93)$. The median percentage of values in therapeutic range using the traditional method was also not significantly higher in the pharmacist-managed group compared to the cardiologist-managed group ( $41.9 \%$ versus $36.8 \%, \mathrm{p}=0.93)$. Conclusion: Warfarin management in a pharmacist-managed group compared to a cardiologist-managed group provided similar care. Differences in time in therapeutic range and median values in therapeutic range were not found between the pharmacist-managed group and cardiologist-managed group. These results describe and provide support that pharmacist-managed anticoagulation of pediatric cardiology patients is safe and effective in the outpatient setting.
\end{abstract}

Keywords Anticoagulation, Warfarin, Pediatrics, Cardiology, Vitamin K Antagonists (VKA), International Normalized Ratio (INR), Pharmacist-managed, Usual Care

\section{Introduction}

Anticoagulation recommendations for pediatric patients vary based on indication and age. Unfractionated heparin (UFH), low molecular weight heparin (LMWH), and vitamin $\mathrm{K}$ antagonists (VKA) have been studied in the pediatric population [1]. In many pediatric subsets, LMWH is preferred due to decreased need for monitoring, fewer drug-drug and drug-food interactions, decreased risk of heparin induced thrombocytopenia, and less interpatient and intrapatient variability $[2,3]$. Despite these advantages, UFH and VKA maintain a place in therapy. Indications for warfarin in pediatric patients include venous thromboembolism (VTE), Fontan surgery, ventricular assist devices (VADs), homozygous protein $\mathrm{C}$ deficiency, Factor V Leiden, sinus or cerebral vein thrombosis, congenital heart disease, valve replacements, and arterial ischemic stroke (AIS) [2].

Warfarin is a vitamin $\mathrm{K}$ antagonist that inhibits coagulation factors II, VII, IX, and $\mathrm{X}$ as well as protein $\mathrm{C}, \mathrm{S}$, and $Z[1,5]$. The international normalized ratio (INR) is used to monitor the safety and efficacy of warfarin. According to the CHEST 2012 Guidelines, in children taking a VKA, the target INR should be 2.5 with a goal range of 2.0 to 3.0 for most indications [2]. The CHEST guidelines suggest a higher INR goal of 2.5 to 3.5 for certain patients, including those with a VAD, mechanical mitral valve, or an aortic bileaflet mechanical valve with risk factors. Provider-specific goals may be implemented to better meet the needs of the patient and clinical circumstances. It is recommended to monitor INR using point of care testing when it is available as simplified testing may improve the outcomes of patients receiving warfarin [2]. 
Management of warfarin can be cumbersome in the pediatric population. Challenges include drug-drug interactions, food-drug interactions, lack of a liquid dosage form, inconsistency in dietary intake of Vitamin K, genetic polymorphisms, frequent INR monitoring, and compliance issues $[1,2,5]$. Vitamin K-dependent coagulation factors are decreased in neonates and infants [2]. Other challenges in infants include variation between vitamin $\mathrm{K}$ content in breast milk and formula and higher weight-based warfarin dosing requirements [2]. Therefore, warfarin is not recommended in infants unless the patient has a mechanical valve. In light of these challenges, warfarin management in pediatric patients requires close monitoring to maintain therapeutic levels and avoid adverse events [1].

Close monitoring of INR values can be evaluated by two different approaches including the traditional method and Rosendaal method. The traditional method utilizes the percentage of values in therapeutic range. The Rosendaal method uses a linear model to assess time in therapeutic range (TTR). The traditional method does not take into consideration the period of time between each INR result; instead, it only provides insight as to what the INR was at the specific time of testing. The Rosendaal method is better correlated with bleeding and thromboembolic events $[4,6]$. For this reason, TTR is considered to be a more reliable representation of the quality of anticoagulation control [6].

To our knowledge, literature describing the utility of a pharmacist in the management of pediatric anticoagulation is limited. However, in the adult setting, studies support pharmacist-managed anticoagulation in the general adult population and in specific populations including patients with left ventricular assist devices and older adults $[7,8,9$, 10]. Bishop and colleagues [7] concluded in patients with left ventricular assist devices, pharmacist-managed anticoagulation demonstrated increased time in therapeutic range compared to usual care and did not find a difference in major bleeding events $(p=0.026)$. In a 2011 retrospective cohort study, Young and colleagues [8] demonstrated increased time in therapeutic range for pharmacist-managed anticoagulation compared to physician-managed over a 17 -month period $(\mathrm{p}<0.0001)$. Patients spent less time below the critical threshold (INR < 1.5 ) and more time above the critical threshold (INR $>5$ ) in the pharmacist-managed group compared to the physician-managed group $(p<0.0001)$ [8]. In 2005, Witt and colleagues [9] published a retrospective observational cohort study that demonstrated an increased amount of time in therapeutic range in the clinical pharmacy anticoagulation services (CPAS) group compared to the usual care group $(\mathrm{p}<0.001)$. The percentage of INR values $\geq 4.0$ or $\leq 1.5$ was decreased in the CPAS group compared to usual care $(p<0.001)$ [9]. Poon and colleagues [10] demonstrated fewer thromboembolic events in the pharmacist-managed group compared to the conventional care group $(\mathrm{p}=0.01)$ despite similar outcomes in the percentage of therapeutic INR values between groups. Although these studies indicate statistically significant differences in the pharmacist-managed group compared to usual care for adult patients, our study describes pharmacist-managed anticoagulation in the pediatric setting. Anticoagulation in the pharmacist-managed group (RXM) is compared it to the cardiologist-managed group (MDM) in this study.

\section{Materials and Methods}

\section{Study Design and Oversight}

This is a single-center, retrospective, quality-improvement study conducted at a children's hospital within a large academic medical center in the United States. It was designed to evaluate the efficacy of a pharmacist-managed warfarin service when compared to a cardiologist-managed warfarin service. A protocol that approved by the institution's Pharmacy and Therapeutics Committee guided dose adjustments and monitoring in the pharmacist-managed group. The protocol was approved on December 18, 2014. Pharmacist management of pediatric anticoagulation was fully transitioned after the approval of the protocol. Written informed consent and IRB approval were not required due to the quality-improvement design of the study.

\section{Study Patients}

Patients who were managed by cardiologists, pharmacists, or both groups for three months or more were included. Patients had to be appropriate candidates for anticoagulation with warfarin. Patients included in the pharmacist-managed group were referred by a pediatric cardiologist with an identified INR goal.

\section{Study End Points}

The primary objective of the study was to evaluate the percentage of time in therapeutic range in the pharmacist-managed group compared to the cardiologist-managed group using the Rosendaal method. Secondary objectives included determining the percentage of INR values in therapeutic range using the traditional method, the number of INR measurements obtained, percentage of critical INR values, and the incidence of major and minor adverse events in the pharmacist-managed group compared to the cardiologist-managed group. Critical INR values were defined as $<1.5$ or $>5$. Minor adverse events included epistaxis, minor bruising, gum bleeding, and delayed cessation of bleeding after lacerations. 


\section{Statistical Analysis}

Safety and efficacy was evaluated for all patients followed by pharmacists or cardiologists for greater than three months. A p-value of 0.05 or less was considered statistically significant. All statistical analyses were performed with the use of R 2.14.0 software. Descriptive statistics were utilized and reported including the median and interquartile range. A t-test was conducted to analyze continuous variables. Power of the analysis is limited due to the small sample size.

\section{Results}

\section{Study Population}

A total of fourteen patients were included. The pharmacist-managed group included twelve patients. There were seven patients in the cardiologist-managed group. Five patients were included in both the pharmacist-managed group and the cardiologist-managed group, seven patients were managed by pharmacists only, and two patients were managed by cardiologists only (a total of fourteen patients). Patients in the cardiologist-managed group were included from March 1, 2013 to September 1, 2014. Patients in the pharmacist-managed group were included from December 31, 2014 to June 30, 2016. A washout period occurred from September 1, 2014 to December 31, 2014, which eliminated inclusion of data during periods of overlapping care between the two groups until pharmacist-managed anticoagulation was fully implemented.

In regards to demographic characteristics (Table 1), most patients were male, receiving anticoagulation for a mitral valve replacement, and had a cardiologist-specified INR goal of 3 to 4 . The median age at the end of the study period was 13 years.

Table 1. Characteristics of the patients according to study group

\begin{tabular}{|c|c|c|}
\hline Baseline Characteristics & $\operatorname{MDM}^{\mathbf{a}}(\mathbf{n}=7)$ & $\operatorname{RXM}^{b}(n=12)$ \\
\hline \multicolumn{3}{|l|}{ Demographics } \\
\hline Male, n (\%) & $7(100)$ & $9(75)$ \\
\hline Median age at end of study, years & 13 & 13 \\
\hline \multicolumn{3}{|l|}{ Indication, $\mathbf{n}(\%)$} \\
\hline Mitral valve replacement & $6(85.7)$ & $7(58.3)$ \\
\hline Aortic valve replacement & $0(0)$ & $1(8.3)$ \\
\hline Aortic and mitral valve replacement & $0(0)$ & $1(8.3)$ \\
\hline Kawasaki disease & $1(14.3)$ & $0(0)$ \\
\hline Factor V Leiden & $0(0)$ & $1(8.3)$ \\
\hline Fontan procedure following stroke & $0(0)$ & $1(8.3)$ \\
\hline Protein S deficiency & $0(0)$ & $1(8.3)$ \\
\hline \multicolumn{3}{|l|}{ INR Goal, n (\%) } \\
\hline 2 to 3 & $0(0)$ & $1(8.3)$ \\
\hline 2 to 2.5 & $0(0)$ & $1(8.3)$ \\
\hline 2.5 to 3.5 & $2(28.6)$ & $2(16.7)$ \\
\hline 3 to 4 & $5(71.4)$ & $7(58.3)$ \\
\hline Other & $0(0)$ & $1(8.3)$ \\
\hline
\end{tabular}

a: MDM: Cardiologist-managed group

b: RXM: Pharmacist-managed group 


\section{Efficacy}

For the primary endpoint, the median percentage of time in therapeutic range using the Rosendaal method was $55.5 \%$ (Interquartile range [IQR] 16.1) in the pharmacist-managed group and 54\% (IQR 15.8) in the cardiologist-managed group $(\mathrm{p}=0.93)$ (Figure 1). In terms of secondary endpoints, the median percentage of values in the therapeutic range in the pharmacist-managed group was $41.9 \%$ (IQR 20.9) compared to 36.8\% (IQR 19.9) in the cardiologist-managed group $(\mathrm{p}=0.93)$ (Figure 2). The median number of INR measurements per patient was 36 (IQR 22.8) in the pharmacist-managed group compared to 28 (IQR 14.5) in the cardiologist-managed group $(\mathrm{p}=0.06)$. The number of supratherapeutic critical INR values in the pharmacist-managed group was 18 (IQR 4.5) compared to 12 (IQR 7.8) in the cardiologist-managed group $(p=0.81)$. The number of subtherapeutic critical INR values in the pharmacist-managed group was 19 (IQR 4.7) compared to 2 (IQR 1.3) in the cardiologist-managed group $(\mathrm{p}=0.17)$ (Table 2).

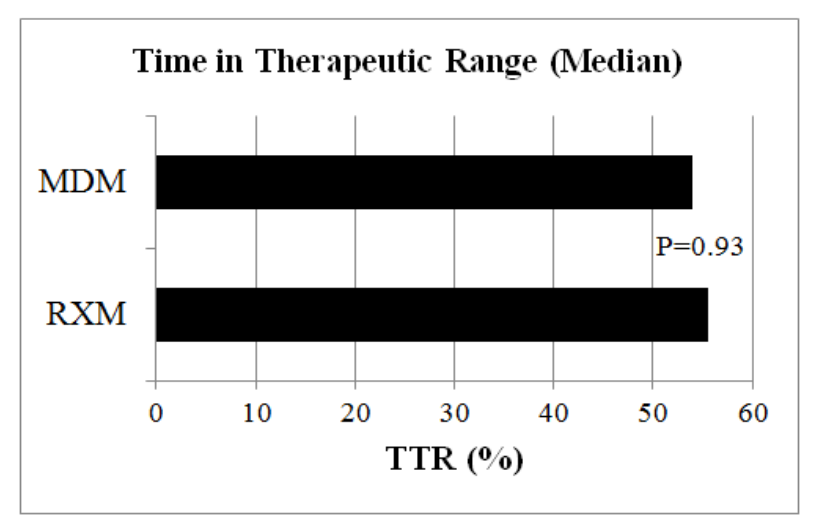

Figure 1. Time in Therapeutic Range (Median)

Figure 1 illustrates the median percentage of time in therapeutic range, $54 \%$ in the physician-managed group compared to $55.5 \%$ in the pharmacist-managed group (using the Rosendaal method).

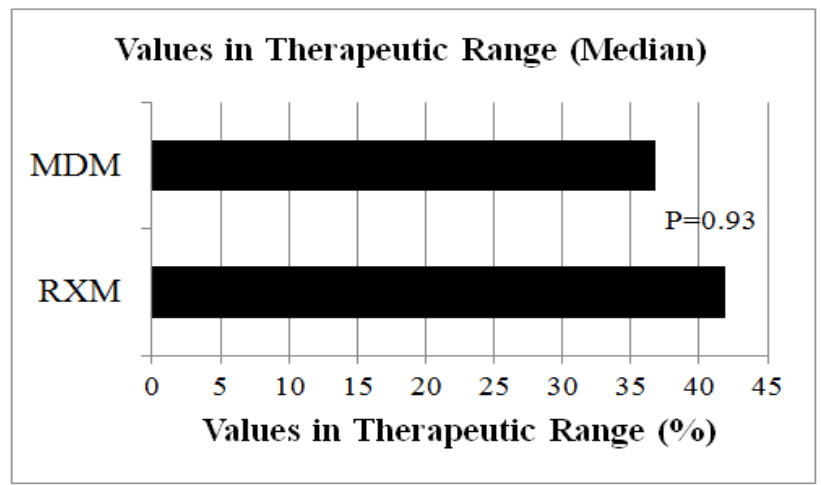

Figure 2. Values in Therapeutic Range (Median)

Figure 2 illustrates the percentage of values in therapeutic range, $36.8 \%$ in the physician-managed group compared to $41.9 \%$ in the pharmacist-managed group (using the traditional method).

\section{Safety}

Adverse events were reported by the patient's caregiver or through direct observation by a health care professional and documented in the patient's medical record. Common adverse events were mild and included epistaxis, minor bruising, gum bleeding, and delayed cessation of bleeding after lacerations. There was one episode of active bleeding that required vitamin $\mathrm{K}$ in the cardiologist-managed group. Of note, this patient was on an antibiotic and inclement weather prevented an INR check. In the pharmacist-managed group there was one episode of thrombosis including the acute right internal jugular and subclavian vein with partial occlusion of the innominate vein. A second patient in the pharmacist-managed group was found to have two infarctions on a brain MRI; however, the timing and etiology of these infarctions were unknown.

Table 2. Results

\begin{tabular}{|c|c|c|c|}
\hline Endpoint & MDM & RXM & P-valu \\
\hline \multicolumn{4}{|l|}{ Primary } \\
\hline $\begin{array}{l}\text { TTR }^{\mathrm{c}}(\text { Rosendaal }), \\
\text { median } \%\left(\mathrm{IQR}^{\mathrm{d}}\right)\end{array}$ & $54(15.8)$ & $55.5(16.1)$ & 0.93 \\
\hline \multicolumn{4}{|l|}{ Secondary } \\
\hline $\begin{array}{l}\text { Values in therapeutic } \\
\text { range, median \% (IQR) }\end{array}$ & $36.8(19.9)$ & $41.9(20.9)$ & 0.93 \\
\hline $\begin{array}{l}\mathrm{INR}^{\mathrm{e}} \text { measurements per } \\
\text { patient, median (IQR) }\end{array}$ & $28(14.5)$ & $36(22.8)$ & 0.06 \\
\hline INR values $>5, \mathrm{n}(\%)$ & $12(7.8)$ & $18(4.5)$ & 0.81 \\
\hline INR values $<1.5, \mathrm{n}(\%)$ & $2(1.3)$ & $19(4.7)$ & 0.17 \\
\hline
\end{tabular}

a: MDM: Cardiologist-managed group b: RXM: Pharmacist-managed group

c: TTR: Time in therapeutic range d: IQR: Interquartile range e: INR: International Normalized Ration

\section{Discussion}

In this single-center, retrospective, quality-improvement study, we found no statistically significant difference in the time in therapeutic range (Rosendaal method), values in therapeutic range (traditional method), number of INR measurements, or critical values between the cardiologist-managed group and pharmacist-managed group during the study period. In the pharmacist-managed group, a standardized schedule for follow-up was utilized based on the approved protocol. This potentially could have increased the amount of INR testing completed by the pharmacist-managed group. As a result, a larger denominator with increased testing could have impacted the outcomes. In the pharmacist-managed group, there 
were fewer INR values $>5$ and more INR values $<1.5$. This could also be accounted for by increased INR testing in the pharmacist-managed group driven by the protocol.

Bishop et al. [7], Young et al. [8], Witt et al. [9], and Poon et al. [10], found statistically significant differences in the pharmacist-managed group compared to usual care when assessing various outcomes such as time in therapeutic range, major bleeding events, thromboembolic events, and INR values, etc. The results of our study in the pediatric population differ from results in the adult setting with no statistically significant differences found in time in therapeutic range, values in therapeutic range, INR measurements per patient, and critical INR values. With limited literature of pharmacist-managed anticoagulation in the pediatric setting, additional studies are necessary to more accurately compare the results of our findings. Future studies should include prospective design, larger sample sizes, and equal groups of patients based on INR goal and indication.

The implications of this new monitoring pathway include several benefits to anticoagulation management in the pediatric setting. Using a Pharmacy and Therapeutics Committee approved protocol, authority is delegated to trained health care providers in the initiation, patient assessment, dose adjustment, and monitoring of warfarin management in the pediatric population. Through the utilization of pharmacists in this initiative, continuity of care for patients receiving warfarin therapy is provided. Additionally, pharmacists are able to assist the physician and the patient in the management of warfarin. Pharmacists can provide education to patients and caregivers in their encounters and encourage personal responsibility for the patient or patient's caregiver (dependent on age). By using a standardized documentation and management approach, recommended follow-up can also foster patient compliance. Overall, pharmacists can impact compliance, continuity of care, and patient (or caregiver) understanding of warfarin therapy through assisting physicians in a standardized approach.

Strengths of this study include a Pharmacy and Therapeutics approved protocol, standardized documentation in the electronic medical record, and close follow-up. This study was limited by a small initial sample size of pediatric patients receiving anticoagulation with warfarin. Several patients in both groups were excluded due to the fact that they were followed for less than three months during the study period. Compliance and difficulties with follow-up (regarding lab draws or inability to reach the caregiver) were also limitations. Prior to the initiation of pharmacist-managed anticoagulation, a standardized note in the electronic medical record was not utilized to document warfarin monitoring and management. Therefore, documentation in the cardiologist-managed group was less standardized and descriptive, which limited data collection in this retrospective study. Another limitation of this study was that patient and caregiver satisfaction were not evaluated.

Currently, the most commonly used anticoagulant in the pediatric setting is low-molecular weight heparin for the treatment of venous thromboembolism [11]. Direct oral anticoagulants (DOACs) including dabigatran (direct thrombin inhibitor), rivaroxaban, apixaban, and edoxaban (factor-Xa inhibitors) have recently gained interest in the pediatric setting based on additional therapeutic monitoring benefits such as minimal need for laboratory monitoring and oral administration $[11,12]$. Despite these advantages, there is limited information on the use of these agents in the pediatric population. Off-label use of these agents is gathered from dosing in the adult setting [12]. In the adult setting, DOACs are not recommended for prophylaxis or treatment of prosthetic valve thrombosis [13]. The agents with the most published data in pediatric patients include rivaroxaban and dabigatran [12]. Currently, there are ongoing studies assessing the pharmacodynamics, pharmacokinetics, safety, and efficacy of DOACs in pediatric patients [12]. Additional studies will need to be conducted in the pediatric setting to determine dosing requirements in obesity, renal insufficiency, or congenital cardiac disease. Until phase III clinical trials with DOACs in the pediatric setting are completed, the use of these agents is severely limited due to lack of safety and efficacy data [11].

In summary, a statistical difference was not found in time in therapeutic range, values in therapeutic range, INR measurements, or critical values between the cardiologist-managed group and pharmacist-managed group during the study period. The results of this study provide preliminary support for pharmacist-managed anticoagulation in the pediatric population given that the care in this group was similar to that in the cardiologist-managed group. This study also serves as a novel contribution to the limited literature regarding the potential role of pharmacists in the management of pediatric anticoagulation. Additional studies are needed to further describe the utility and value of a pharmacist-managed pediatric anticoagulation service.

\section{Acknowledgements}

This abstract has been presented at the American Society of Health-System Pharmacists Midyear Clinical Meeting in Las Vegas, NV on December 5, 2016.

\section{Disclosure}

The authors declare no conflicts or financial interest in any product or service mentioned in the manuscript, including grants, equipment, medications, employment, gifts, and honoraria. 


\section{REFERENCES}

[1] Giglia TM, Massicotte MP, Tweddell JS, et al. Prevention and treatment of thrombosis in pediatric and congenital heart disease: a scientific statement from the American Heart Association. Circulation 2013; 128: 2622.

[2] Monagle P, Chan AK, Goldenberg NA, Ichord RN, Journeycake JM, Nowak-G.ttl U, Vesely SK; American College of Chest Physicians. Antithrombotic therapy in neonates and children: antithrombotic therapy and prevention of thrombosis, 9th ed: American College of Chest Physicians evidence-based clinical practice guidelines. Chest. 2012;141:737S-801S.

[3] Dabbous MK, Sakr FR, Malaeb DN. Anticoagulant therapy in pediatrics. Journal of Basic and Clinical Pharmacy. 2014; $5(2): 27-33$

[4] Rosendaal FR, Cannegieter SC, Van der Meer FJ, Briët E: A method to determine the optimal intensity of oral anticoagulant therapy. Thromb Haemost. 1993; 69: 236-239.

[5] Malec L, Young G. Treatment of Venous Thromboembolism in Pediatric Patients. Frontiers in Pediatrics. 2017; 5:26.

[6] Entezari-Maleki T, Dousti S, Hamishehkar H, et al. A systematic review on comparing 2 common models for management of warfarin therapy; pharmacist-led service versus usual medical care. The Journal of Clinical Pharmacology. 2015; 20(20):1-15.

[7] Bishop MA, Streiff MB, Ensor CR, et al. Pharmacist-managed international normalized ratio patient self- testing is associated with increased time in therapeutic range in patients with left ventricular assist devices at an academic medical center. ASAIO J 2014; 60(2):193-8.

[8] Young S, Bishop L, Twells L, et al. Comparison of pharmacist-managed anticoagulation with usual medical care in a family medicine clinic. BMC Family Practice 2011; 12(88):1-7.

[9] Witt DM, Sadler MA, Shanahan RL, et al. Effect of centralized clinical pharmacy anticoagulation service on the outcomes of anticoagulation therapy. Chest. 2005 May; 127(5): 1515-22.

[10] Poon IO, Lal L, Brown EN, Braun UK. The impact of pharmacist-managed oral anticoagulation therapy in older veterans. J Clin Pharm Ther. 2007 Feb; 32(1):21-9.

[11] Oldenberg NA, Takemoto CM, Yee DL, Kittelson JM, Massicotte MP. Improving evidence on anticoagulant therapies for venous thromboembolism in children: key challenges and opportunities. Blood. 2015 Dec 10; 126(24): 2541-7.

[12] Von Vajna E, Alam R, So T-Y. Current Clinical Trials on the Use of Direct Oral Anticoagulants in the Pediatric Population. Cardiology and Therapy. 2016; 5(1):19-41.

[13] Nishimura RA, Otto CM, Bonow RO, Carabello BA, Erwin JP 3rd, Fleisher LA, Jneid H, Mack MJ, McLeod CJ, O'Gara PT, Rigolin VH, Sundt TM 3rd, Thompson A. 2017 AHA/ACC focused update of the 2014 AHA/ACC guideline for the management of patients with valvular heart disease: a report of the American College of Cardiology/American Heart Association Task Force on Clinical Practice Guidelines. Circulation. 2017; 1-60. 